\title{
Patient assessment of treatment following knee injury
}

\author{
W. George Kernohan PhD, Francis J. Dodd MSc*, \\ K. Elizabeth Dowey MRCP† and Lisa A. McConnell \\ School of Clinical Medicine, The Queen's University of Belfast, UK, *Department of Mathematics, Faculty of \\ Informatics, The University of Ulster, UK, + Sports Injury Clinic, Belfast City Hospital, UK, and $\ddagger$ Faculty of \\ Medicine, Birmingham University, UK
}

\begin{abstract}
A survey of 100 sports-injured cases was carried out to elicit patients' personal assessment of their treatment and to investigate the utility of a typical database system for recording and analysis. The cases were limited to knee injuries, with a high proportion arising from football of various types and interesting light was thrown on several possible contributory factors.
\end{abstract}

Keywords: Knee-injuries, football, patient assessment, database

It is the knee joint which provides orthopaedic surgeons with the greatest problem in respect of diagnosis. Not without valid reason has the knee been labelled a 'diagnostic minefield' ${ }^{1}$ and few other joint injuries cause athletes such concern ${ }^{2}$. With the recognized maturity of the sports injury clinic set up at Belfast City Hospital in $1981^{3}$, a questionnaire survey of 100 knee injuries was initiated to elicit patients' personal assessment of their treatment. It was felt that computers might assist the operation and assessment of the clinic, and in particular the efficacy of treatment. More general questions that might be asked are: 'What are the most common injuries in each sport?', 'What provisions could reduce these?', 'What provisions could minimize their effects?'. As a practical, economic exercise a database system was set up. The principal aims were: (1) to obtain patients' views of their injury, subsequent treatment and final outcome; (2) to investigate the utility of a typical database system in this field and to discover the problems that might arise in the setting up of a more comprehensive database; (3) to determine the necessity or usefulness of the information that might be held.

The data for this system were limited to knee injuries, the most common injury seen at the clinic, and were voluntarily provided by former recent patients, 100 of whom were circularized with a

Address for correspondence: Dr G. Kernohan, University Department of Orthopaedic Surgery, Musgrave Park Hospital, Belfast BT9 7JB, Northern Ireland, UK

(C) 1993 Butterworth-Heinemann Ltd 0306-3674/93/020131-04 questionnaire. Of the 100 so questioned, 65 replied with completed questionnaires. It is impossible totally to exclude bias in the group of respondents arising through seasonal factors in addition to the more obvious ones, e.g. willingness to fill in forms and answer questions.

Treatment had not always been completed and the answers to some questions, particularly those relating to their assessment of treatment, were therefore to some extent preliminary.

\section{A typical database system}

The most promising type of system for holding the data from this type of survey is a relational database which provides for more flexibility in ad hoc queries than other types, e.g. hierarchical databases. In this case, the readily available VAX Datatrieve (Digital Equipment, Maynard, Massachusetts, USA) ${ }^{4}$ was used. The statistics available in version V4.1 of Datatrieve were limited to simple measures, e.g. the mean, variance, etc. Sophisticated features such as correlation coefficients would be desirable in a practical system as a simpler alternative to the facility for exporting to a statistical package. A mechanism for applying contingency tables to investigate links between several factors would be particularly useful. Unfortunately the frequencies in many of the subcategories cannot be guaranteed to be greater than five for consistent use of the $\chi^{2}$ test. Future developments in statistical database packages are eagerly awaited.

For convenience many fields (data items) in the database were coded with a single letter, e.g. 'standard of sport', A = international, B = interprovincial, etc. Where such fields relate to diagnosis and treatment and are selected on an ordinal scale (i.e. ranked in some way, in contrast to a scale which is purely nominal or categorical) the $\chi^{2}$ test can be used to compare them with, or 'correlate' them against, other such scales and other interval scales ${ }^{5}$. For example, the consequences of the injury might be assessed by the deterioration in playing standard (i.e. the difference between standard before and standard after, letters from $A$ to D), working hours lost as a result of the injury, and reduction in weekly playing hours since the injury. These fields might be matched 
against type of injury or type of treatment, both one letter fields (ranked to some extent) by gravity. While it would appear that more information could be extracted by using only numerical coding, the handling of letters comes much more naturally to most minds and a numeric scale may often be perceived misleadingly to have an interval quality.

\section{Data considerations}

Although quickly obtained, information collected by questionnaire survey has some limitations. In relation to the sporting and total populations it is neither completely random nor a systematic sample; the absence of control groups excludes generalizations on the sporting population as a whole. Data collected in a questionnaire survey tends to be general rather than technical (where, for example, diagnosis is involved), and is inevitably subjective. On the other hand, the data should be more objective and free of observer bias when compared with those collected via interviews.

It is first worth mentioning the population differences between the patients with knee injury used in the database and the patients seen by the clinic. The ratio of men to women in the database was similar; $4: 1(52 / 13)$ as against $5: 1(885 / 173)$ for patients generally. (The $95 \%$ confidence interval (c.i.) for the difference includes zero: -0.14 to 0.06 , i.e. no significant difference.) Running has been reported as the principal sport involved in sporting injuries generally $\left(43 \%{ }^{3}\right)$ with football a considerable distance behind $(29 \%)$; in this survey, however, football (soccer) was top with 24 cases $(37 \%)$ with a further 12 cases of other types of football and only one case (less than $2 \%)$ for running $(95 \%$ c.i. for difference in proportion of running-related knee injury 0.37 to 0.45 and for football-related injury 0.15 to 0.40 ).

This provided evidence for some secondary lines of possible future research and also indicates that injuries of specific types, and to specific anatomical sites, might be sport dependent in ways that are not at all obvious.

The predominance of football (soccer) as the incidental sport might also be reflected in the higher proportion of international players injured, 4/65 in the sample against $16 / 1058$ at the clinic. However, four is too small a number on which to base conclusions relating to recovery rates, although top-level or competitive athletes do have significantly better healing results than other patients ${ }^{2}$. Other differences between the various types of football (soccer, rugby and Gaelic) and other sports proved to be of further interest and are discussed below.

\section{The data}

The fields, i.e. data items, held in the database were non-technical because of the method of collection. They are grouped under the headings: personal, prevailing conditions, the injury itself, the treatment, the patient's assessment of the treatment, and the consequences of the injury. Personal information was of little interest in relation to the aspects of the database under discussion here, e.g. name, sex, date
Table 1. Showing the body mass indices for the study group

\begin{tabular}{lrrrrrrrrrr}
\hline$B M I$ & $<20$ & 20 & 21 & 22 & 23 & 24 & 25 & 26 & 27 & $>27$ \\
\hline Men & 2 & 4 & 2 & 3 & 14 & 7 & 8 & 5 & 4 & 3 \\
Women & 2 & 4 & 1 & 2 & 1 & 2 & 1 & - & - & - \\
\hline
\end{tabular}

of birth, age at time of injury. Each record (i.e. data relating to a single instance of injury) must be uniquely identified by a 'key field' to permit easy access for, e.g. error checking. An artificial field might be used for this but a composite field of date of injury and date of birth proved sufficient for the system. The height and weight were given in feet/inches and stones/pounds (usually) since the imperial scales are still more familiar than metric to respondents. But a multiplication (by 68.354) permits conversion of the body mass index (BMI) from stones per square foot to the more usual $\mathrm{kg} \mathrm{m}^{-2}$. It has been pointed out ${ }^{6}$ that this formula used for the the BMI may be a special case of weight/height ${ }^{p}$ where $p$ is dependent on the population under scrutiny, a value of 2 for $p$ has been shown to be accurate for the UK.

The BMI was noticeably lower for women: mean (range) 21.46 (18-26); than men: mean (range) 23.94 (19-39); see Table 1. The highest BMI was for a cricketer. Although $12(23 \%)$ men would be classed as overweight (BMI > 25) as against no women on this scale, nothing can be deduced because of the small number of women. The BMI tends in any case to be slightly lower for women than men.

\section{Prevailing conditions}

Data classified under possible contributory factors include the sport being played at the time of injury, the warm-up (or absence thereof) before playing, and time of the injury within the game (e.g. five at the beginning, 37 in the middle, and 21 towards the end). Footballers appear to be injured towards the end of the game, 13 of 36 against 8 of 29 for other sports, though the difference is not significant (95\% c.i. -0.14 to 0.31 ). This might be caused by exhaustion leading to accident or a speeding up in the closing minutes in games of fixed length (such as football) which may not be found in other games limited by the score.

The immediate cause of injury, e.g. a twist (37 cases or $57 \%$ ), a fall (6 cases or $9 \%$ ), contact with others ( 7 cases or $11 \%$ ) could be investigated as possible contributory factors to, or influences on, the injury. Although relationships between an inadequate warm-up and other data fields (e.g. relating to gravity, consequences and type of injury) are generally recognized, accurate quantification of this might be extracted from more complete data.

\section{Nature and extent of injury}

The injury itself is characterized by factors other than anatomical site, diagnosis (pulled muscle, ligament damage, tendinitis, etc.) and extent (pain when running, difficulty with walking, etc.). Its speed of 
appearance or development (suddenly, within hours, days, weeks) may be significant both with regard to its nature and its treatment. The most frequent type of injury was ligament damage (26 cases or $40 \%$ ), followed by cartilage trouble (18 cases or $28 \%$ ), with other causes trailing well behind, e.g. tendinitis with 7 cases $(11 \%)$.

Here again differences showed up between football and other sports: ligaments were at risk in football, 18 against eight for other sports. Similarly, injuries were significantly more sudden in football (35/36 against $20 / 29,95 \%$ c.i. 0.11 to 0.46 ), more likely to be caused by a twist (22/36 against $15 / 29,95 \%$ c.i. -0.15 to 0.34 , not significant) or, less commonly, by physical contact (5/36 against $1 / 29,95 \%$ c.i. -0.03 to 0.24 , not significant), and have more immediate consequences, 22 completely immobilized against five for other sports $(95 \%$ c.i. 0.23 to 0.65$)$.

\section{The treatment}

The treatment, one of the most important elements in the survey, is perhaps the most complex. A first (or on the spot) treatment is likely to precede the clinical treatment, particularly when the patient has been referred, and there may be a significant time lag between the two. Although 20 patients attended the clinic within 3 days, the average time to the clinic was 63 days; this is distorted by a minority (eight patients) who did not arrive until over 6 months had elapsed, including one patient who waited 600 days (too late for effective treatment of his particular injury).

A comparison of the patients referred by a casualty centre $(25$ cases or $38 \%)$ with those referred by a general practitioner (34 cases or $52 \%$ ) might prove significant. Not surprisingly, those referred by casualty tended to be more severe (Table 2). However, the time taken to arrive at the clinic was only slightly less (50 against 74 days). That footballers were slightly more likely to be referred by casualty $(15 / 36$ against $10 / 29,95 \%$ c.i. -0.16 to 0.31 , not significant) may be caused by the times (usually weekend) at which football is played.

The type of referral might also be reflected in the time lapses between injury and diagnosis/final outcome. In practical terms, if a quick diagnosis enhances the chances of successful treatment, then the possibility that the clinic be held more frequently than once weekly might be considered. Such a decision would, of course, have to take into account more general considerations, not least the resources available.

Table 2. Type of referral (from casualty or from family doctor) against injury severity

\begin{tabular}{lccccc}
\hline & $\begin{array}{c}\text { Unable } \\
\text { to } \\
\text { walk }\end{array}$ & $\begin{array}{c}\text { Walk } \\
\text { with } \\
\text { difficulty }\end{array}$ & $\begin{array}{c}\text { Pain } \\
\text { when } \\
\text { running }\end{array}$ & $\begin{array}{c}\text { Working Reduction } \\
\text { hours } \\
\text { lost }\end{array}$ & $\begin{array}{c}\text { Red playing } \\
\text { hours }\end{array}$ \\
\hline $\begin{array}{l}\text { Casualty } \\
\text { General } \\
\text { practitioner }\end{array}$ & 9 & 14 & 2 & 27 & 5 \\
\hline
\end{tabular}

The possibility of two distinct treatment times as mentioned above is further complicated by multiple treatments. For example, 47 patients were initially recommended merely to rest and let time take effect; of these only 11 were given the same advice at the clinic. While this may seem alarming in that 15 of these patients subsequently required arthroscopy or surgery of another sort, it is attenuated by the recommendation of physiotherapy (a treatment compatible with rest) for 16 other patients. Even the 15 patients for surgery might be misleading - a minor misunderstanding of the question might have led some patients who had been advised to rest until fuller treatment was available to select this answer to the question on initial treatment. Similarly, four of the five patients initially recommended pain-killers were subsequently 'demoted' to rest or physiotherapy - any pain might have vanished by the time they attended the clinic.

\section{Patients' assessment of treatment}

The complexities of treatment carry over into the patients' assessment of their treatment. The questionnaire contained questions on the effectiveness of physiotherapy as distinct from the overall treatment. Only three of the 24 patients to whom physiotherapy was recommended as the main treatment showed dissatisfaction, although five of these treatments were incomplete. This level of satisfaction is reflected by patients receiving physiotherapy as a subsidiary treatment; in the survey group as a whole, only six of 35 were dissatisfied, with 23 indicating positive satisfaction. The type of referral and sport practised appeared to make no appreciable difference. More complex treatment data (with questions similar to those on physiotherapy on all of the individual aspects of treatment) should greatly improve this, and the next, function of the database.

The expressed assessment by patients of their overall treatment contrasts with this: on a scale of 1 to $5,25(38 \%)$ expressed themselves as generally dissatisfied, $19(29 \%)$ undecided and $23(35 \%)$ generally satisfied. The high level of dissatisfaction with treatment would at first sight suggest that improvements in this area might be productive. However, it is possible that the treatment at the clinic is in fact satisfactory in the light of the observations of Simpson et al. ${ }^{7}$, in particular that patient and doctor disagree on $50 \%$ of diagnoses. Patients' differing interpretations of the scale is reflected in the low measure of neighbourhood consensus of satisfaction, namely $0.46^{8}$.

This is the aspect of the database prone to subjectivity, and satisfaction with treatment can improve simply by better communication ${ }^{9}$. The neglect of this area often leads to unrealistic expectations of the medical profession by the public at large. This, together with the obvious subjectivity of many answers under this heading, is borne out by the comments in the section provided on the questionnaire which, to a large extent, contradicts the unfavourable assessment of treatment. For example, of those who gave a score of 3 (the mid-point of the scale), one admitted that rest had improved the 
injury 'immensely'. The same score (3) was awarded by a case that reported 'no significant improvement'.

Other cases seemed to realise that their own impatience had not helped - the clinic was expected to remove the need for rest and time. In few cases did there appear to be genuine dissatisfaction with the clinic.

\section{Consequences of the injury}

In contrast to the subjective assessments of treatment, the consequences of the injury are perhaps the most measurable elements in the database, this unfortunately was the area most affected by incomplete treatment or the passage of time.

As mentioned previously, the standard before and the standard after the injury cannot be regarded as interval scales, i.e. the difference between an international and an interprovincial player is not necessarily equal to that between a club and a friendly player. Even so the working hours lost, and reduction in playing hours since the injury are and might usefully be correlated against the possible contributory factors listed above. It is impossible from the survey data to distinguish between reductions through the injury and those resulting from other factors.

This latter may partly explain the striking reduction in the average hours played from $6 \mathrm{~h}$ to $1 \mathrm{~h}$ weekly. In more detail, $15(23 \%)$ patients were unaffected in this respect against $40(62 \%)$ who gave up sport entirely. The 40 include 31 who discontinued a weekly sport ration of $4 \mathrm{~h}, 21$ of $6 \mathrm{~h}, 9$ of $10 \mathrm{~h}$, and even 2 of $20 \mathrm{~h}$. Unless reflecting temporary conditions, these figures present a depressing picture of the effects of sports injuries. Proponents of sporting activities would hope that age and injury would cause transfers to less strenuous sports rather than complete cessation.

The questionnaire also asked for other sports played. An ambiguity in this question left it unclear that before the time of the injury rather than after was implied here. In at least one case (where the answer was 'leg extensions'), there were implications that the patient had taken up the exercise as a result of the injury, possibly on physiotherapeutic advice.

If a single database system is to be used to hold clinical information, current patients must be excluded from certain types of final assessment queries. Treatment evaluation, however, would be a fundamental aspect of any future system.
If a relational database is adopted in any future system, a front-end facility would be essential in order to simplify standard queries and encourage non-computer specialist use. This would also provide for the validation and standardization of data input so avoiding input problems, e.g. mixing upper and lower case, typing errors, etc.

In this study, the representation of certain sections of the population, e.g. the young and the old, was too small for statistical analysis. Nevertheless, the exercise is seen as having been worthwhile in view of both the experience gained and the results obtained from the system.

The more successful the treatment of sports injury, the less likely the patient is to reappear at the clinic. Patient assessment of treatment can be obtained only by methods such as the questionnaire survey. It is impossible to eliminate incidental factors which might lead to bias, e.g. those dissatisfied with treatment might be more likely to respond in order to vent their feelings. The possibility of obviating this problem in a more permanent database is currently being examined, e.g. questionnaires might be given immediately after completion of treatment and again, after a period of, say, 6 months. Certainly the open comments section of the questionnaire often proved its worth by shedding valuable light on other answers in the questionnaire.

\section{References}

1 Helal B, Chen SC. Arthroscopy of the knee. Br J Hosp Med 1976; 583-95.

2 Kannus P, Jarvinen M. Role of sports in etiology and prognosis of surgically treated acute knee ligament injuries. Int J Sports Med 1986; 7: 39-43.

3 Dowey KE. Preliminary report - sports injury clinic. Ulster Med J 1984; 53: 88-92.

4 VAX Datatrieve Handbook, Maynard, Massachusetts, USA Digital Equipment Corporation, 1987.

5 Siegel S, Castellan NJ. Nonparametric Statistics for the Behavioural Sciences. London, UK: McGraw Hill, 1988.

6 Knight IB, Elridge J. The Heights and Weights of Adults in Great Britain, Report of a Survey Carried Out on Behalf of the Department of Health and Social Security Covering Adults Aged 16-64. London, UK: HMSO, 1984.

7 Simpson M, Buckman R, Stewart $M$ et al. Doctor patient communication: the Toronto Consensus Statement. Br Med I 1991; 303: 1385-7.

8 Donegan HA, Dodd FJ. An analytical approach to consensus. Appl Math Lett 1991; 4: 21-4.

9 Ley P. Satisfaction, compliance and communication. $\mathrm{Br} J$ Clin Psych 1982; 21: 241-54. 